and the anomalous SVC to left atrium vessel. The proximal and distal open ends of the SVC above and below the pulmonary vein orifices were closed by direct suturing with 6/0 Prolene suture (Ethicon, Inc, Somerville, NJ), thus allowing the anomalous veins to drain to the left atrium through the anomalous vessel from the SVC to the left atrium. The SVC was reconstituted by direct anastomosis. The patent foramen ovale was closed by direct suture (Figure 1, B). Weaning from cardiopulmonary bypass was accomplished without problems. Postoperative echocardiography showed no shunt at the SVC or the atrial level, good biventricular function, and no obstruction of pulmonary venous return. The patient was transferred to the cardiac intensive care unit and was discharged home on the 4 th postoperative day.

\section{DISCUSSION}

This is the second case report in the English-language literature to describe isolated PAPVC with concomitant anomalous connection of the SVC to the left atrium. ${ }^{4}$ The surgical repair consisted of a direct complete repair. The unobstructed PAPVC to the SVC could be directed from the SVC to the left atrium through the auxiliary connection. The remainder of the procedure consisted of a direct SVC to right atrial connection without the need for a removal of the tip of the atrial appendage.

Preoperative diagnosis of isolated PAPVR may be difficult because of lung overlying the pulmonary veins and the
SVC. Where there is particular doubt, magnetic resonance imaging (MRI) may be considered for supplementary diagnosis. Although standard evaluation with T1-weighted spin echo images can delineate the exact anatomy, cine-MRI with flow-sensitive sequences may reveal information about the exact size, course, and drainage of the PAPVR. Furthermore, 3-dimensional imaging can be created with magnetic resonance angiography. ${ }^{3}$ If the echocardiographic images are easy to obtain and there is no urgent need, however, MRI is usually not preferred in the younger patient population because of the risks associated with sedation and intubation. Cardiac catheterization is rarely necessary.

In conclusion, even if an accurate preoperative evaluation with echocardiography, MRI, or both has been performed, careful understanding of the entire cardiac anatomy should be achieved intraoperatively in patients with PAPVC to allow application of an appropriate surgical repair.

\section{References}

1. Hughes C, Rumore P. Anomalous pulmonary veins. Arch Pathol. 1944;37:364-6.

2. Healey JE Jr. An anatomic survey of anomalous pulmonary veins: their clinical significance. J Thorac Surg. 1952;23:433-44.

3. Geva T, Van Praagh S. Anomalies of the pulmonary veins. In: Allen HD Driscoll DJ, Shaddy RE, Feltes TF, eds. Moss and Adams' heart diseases in infants, children, and adolescents. 7th ed. Philadelphia: Lippincott Williams \& Wilkins; 2008. p. 761-92.

4. Beck C, Wessel A, Yelbuz TM, Bertram H. Images in cardiovascular medicine Unusual case of anomalous pulmonary venous return with left atrial to systemic venous shunt. Circulation. 2006;113. e840-1.

\title{
Cardiac extension of an intravascular leiomyomatosis 43 years after hysterectomy
}

\author{
François Cornélis, MD, ${ }^{\mathrm{a}}$ Geneviève Belleannée, MD, ${ }^{\mathrm{b}}$ and Mathieu Lederlin, MD, ${ }^{\mathrm{a}}$ Pessac, France
}

Intravenous leiomyomatosis is a rare benign smooth muscle tumor most frequently seen in premenopausal women. Extension through the inferior vena cava into the right heart chambers can cause valve obstruction, leading to cardiac insufficiency and death. Definitive treatment is surgical; however, recurrence from incomplete excision may

\footnotetext{
From the Departments of Thoracic and Cardiovascular Imaging ${ }^{\mathrm{a}}$ and Pathology, Hôpital Haut-Lévèque, CHU Bordeaux, Pessac, France.

Disclosures: Authors have nothing to disclose with regard to commercial support.

Received for publication Feb 1, 2012; accepted for publication March 12, 2012; available ahead of print April 9, 2012.

Address for reprints: Mathieu Lederlin, MD, Department of Thoracic and Cardiovascular Imaging, Hôpital Haut-Lévèque, CHU Bordeaux, 33604 Pessac, France (E-mail: mathieu.lederlin@chu-bordeaux.fr).

J Thorac Cardiovasc Surg 2012;144:e3-5

$0022-5223 / \$ 36.00$

Copyright (c) 2012 by The American Association for Thoracic Surgery

doi:10.1016/j.jtcvs.2012.03.022
}

necessitate reoperation. Intravenous leiomyomatosis should therefore be considered in the differential diagnosis of a middle-aged woman with a right heart tumor.

\section{CLINICAL SUMMARY}

A 75-year-old woman was referred to our institution with swollen legs. She had a history of a hysterectomy and a bilateral ovariectomy at the age of 32 years for a myomatous uterus. Three years before the referral, the patient underwent a surgical resection of a left-sided pelvic tumor that was noted to be a leiomyoma. In the postoperative period, a left internal iliac vein and inferior vena cava thrombosis was diagnosed, and she received anticoagulation therapy for this condition.

Physical examination showed swollen legs without any other abnormality. Cardiac auscultation revealed a tricuspid 

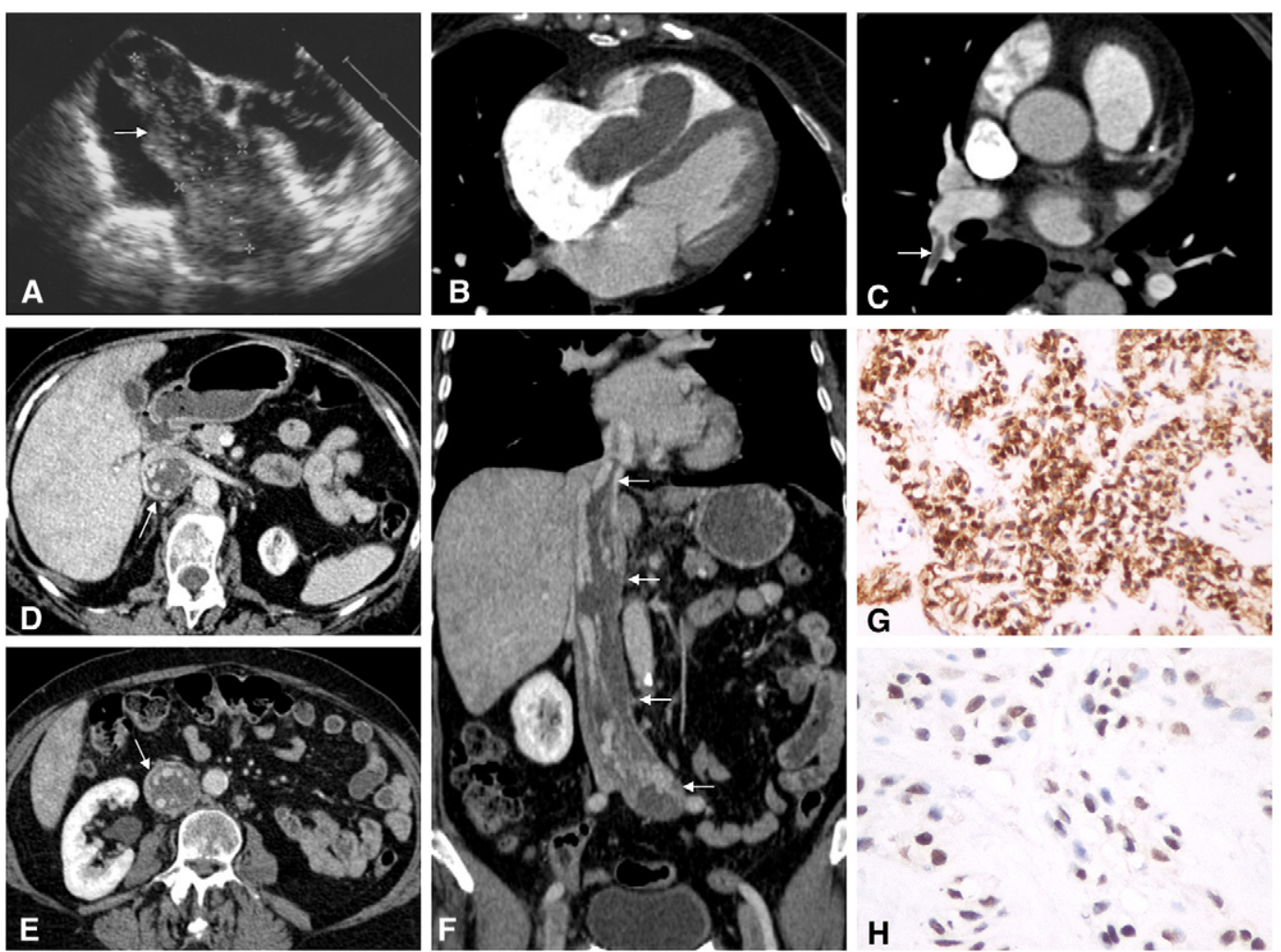

FIGURE 1. A, Transthoracic echocardiogram showing a mobile solid mass extending into the right cardiac cavities (arrow). B, Axial contrast-enhanced chest computed tomography: solid mass extending into the right atrium and ventricle through the tricuspid valve. C, Axial contrast-enhanced chest computed tomography: endoluminal thrombus (arrow) within the right lower lobar artery and its segmental branches. D, Axial contrast-enhanced abdominal computed tomography: enlargement of the inferior vena cava (arrow), which is almost completely occluded by a partly permeable thrombus. E, Axial contrastenhanced abdominal computed tomography: extension of the thrombus into the lower inferior vena cava (arrow). F, Coronal reformation of contrastenhanced computed tomography at chest and abdominal-pelvic levels: thrombus extending into the whole inferior vena cava (arrows) and continuing to the left common iliac vein (lower arrow). G, Microscopic image from the tumor stained with anti-alpha actin antibody (20× magnification) showing smooth muscle infiltration within the tumor. H, Microscopic image from the tumor stained with progesterone receptor antibody ( $40 \times$ magnification) showing cells intensely positive for progesterone receptor.

regurgitation murmur. A 12-lead electrocardiogram showed a sinus rhythm with nonspecific findings, and the chest radiograph appeared normal. Transthoracic echocardiography revealed a mobile mass extending from the inferior vena cava through the right atrium and the right ventricle (Figure 1, A, arrow).

A subsequent postcontrast computed tomographic scan clearly demonstrated a large right atrial mass prolapsing through the tricuspid valve into the right ventricle (Figure 1, B). A small pulmonary embolism was also seen (Figure 1, $C$, arrow). Abdominal and pelvic computed tomographic scans showed a voluminous, free-floating, and elongated thrombus of the entire inferior vena cava (Figure 1,D and $E$, arrows), extending from the left internal iliac vein through the right cardiac cavities (Figure 1, F, arrow). The thrombus was partly repermeabilized and was not adherent to the walls of the vessels and the cardiac cavities.

The cardiac thrombus was surgically removed under conditions of deep hypothermia and circulatory arrest. The patient showed a favorable outcome. Histologic study of the resection piece revealed an intravenous leiomyomatosis characterized by proliferation of typical smooth muscle cells showing positive staining with smooth muscle antibody (Figure 1, $G$ ). The positive staining of the tumor cell nuclei with progesterone receptor antibody (Figure 1, $H$ ) argued strongly in favor of the gynecologic origin of the neoplasm. Rereading of the pelvic tumor excised 3 years previously confirmed the diagnosis of intravascular leiomyomatosis of uterine origin.

\section{DISCUSSION}

Intravenous leiomyomatosis is a rare benign smooth muscle tumor arising from the wall of pelvic veins or from a uterine leiomyoma that has infiltrated a vein. ${ }^{1}$ This proliferation spreads intravenously through uterine and ovarian veins and sometimes into the inferior vena cava and the right cavities. This pathologic entity is most frequently seen in premenopausal women. Clinical onset of the disease depends on the extension of the lesions, but many cases are discovered incidentally. 
Extension to the right heart chambers can cause valve obstruction, leading to cardiac insufficiency and death. Magnetic resonance and computed tomographic angiography are the most sensitive methods to diagnose this pathology and to enable planning of the most appropriate therapeutic strategy. ${ }^{2}$ Treatment consists in a total hysterectomy and bilateral salpingo-oophorectomy, along with removal of as much of the extrauterine tumor as possible. Recurrence can result from incomplete excision and may necessitate further surgical treatment. Because of the presence of estrogen receptors, antiestrogens such as tamoxifen may be given when the tumor is unresectable or to control for incomplete resection or recurrence of the tumor. $^{3}$
In conclusion, intravenous leiomyomatosis should be considered in the differential diagnosis of a middle-aged or older woman with a right-heart tumor. Histopathologic diagnosis must be made because other tumors, such as leiomyosarcomas of the retroperitoneum, may mimic intravascular leiomyomatosis.

\section{References}

1. Politzer F, Kronzon I, Wieczorek R, Feiner H, De Marco LE, Weintraub PR, et al Intracardiac leiomyomatosis: diagnosis and treatment. J Am Coll Cardiol. 1984;4: 629-34.

2. Maraj S, Pressman GS, Figueredo VM. Primary cardiac tumors. Int J Cardiol. 2009;133:152-6.

3. Tierney WM, Ehrlich CE, Bailey JC, King RD, Roth LM, Wann LS. Intravenous leiomyomatosis of the uterus with extension into the heart. Am J Med. 1980;69:471-5.

\title{
Management of postdissection thoracoabdominal aneurysm after previous frozen elephant trunk procedure with the E-vita Open Plus stent-graft
}

\author{
Vito Giovanni Ruggieri, MD, Amedeo Anselmi, MD, Issam Abouliatim, MD, PhD, and \\ Jean-Philippe Verhoye, MD, PhD, Rennes, France
}

The use of a hybrid stent-graft enables the extension of the aortic repair into the dissected descending aorta in patients undergoing aortic surgery through a median sternotomy. The frozen elephant trunk technique has been reported to decrease the rate of aortic reoperation, both open and endovascular, after repair of type A acute aortic dissection. ${ }^{1,2}$ Here we discuss a case of thoracoabdominal aneurysm treated with conventional surgery after a previous frozen elephant trunk procedure with the E-vita Open Plus hybrid endoprosthesis (JOTEC GmbH, Hechingen, Germany).

\section{CLINICAL SUMMARY}

A 33-year-old male patient was admitted with diagnosis of type A acute aortic dissection. The intimal tear was

\footnotetext{
From the Division of Cardiovascular and Thoracic Surgery, Pontchaillou Hospital, Rennes, France.

Disclosures: Authors have nothing to disclose with regard to commercial support. E-vita Open Plus is manufactured by JOTEC GmbH, Hechingen, Germany.

Received for publication Jan 26, 2012; revisions received March 6, 2012; accepted for publication March 16, 2012; available ahead of print April 16, 2012.

Address for reprints: Vito Giovanni Ruggieri, MD, Division of Cardiovascular and

Thoracic Surgery, Centre Hospitalier Universitaire Pontchaillou, 2 Rue Henri le

Guilloux, 35003 Rennes cedex 9, France (E-mail: gianvito_ruggieri@libero.it).

J Thorac Cardiovasc Surg 2012;144:e5-7

$0022-5223 / \$ 36.00$

Copyright (C) 2012 by The American Association for Thoracic Surgery

doi:10.1016/j.jtcvs.2012.03.031
}

localized into the ascending aorta. We performed an emergency David procedure, and the aortic arch was free from intimal tears. The day after surgery, the patient's primary care physician informed us about a diagnosis of Marfan syndrome, which was later confirmed histopathologically. Predischarge computed tomographic scan showed the extension of the dissection down to the abdominal aorta, without aneurysmal dilatation. Twenty-six months later, the patient came back with both worsening dyspnea and dorsal and lumbar pain. Computed tomographic scan revealed aneurysmal dilatation of the aortic arch (maximum diameter, $43 \mathrm{~mm}$ ), of the descending aorta $(56 \mathrm{~mm})$, and of the abdominal aorta $(66 \mathrm{~mm})$. The main visceral vessels emerged from the true lumen. Echocardiography revealed severe mitral regurgitation and moderate systolic dysfunction of the left ventricle. We performed a mitral valve replacement associated with aortic arch replacement and descending aortic stent-grafting according to the frozen elephant trunk technique ${ }^{3}$ with a 24-mm E-vita Open Plus hybrid prosthesis. Three months later, the patient was readmitted for intractable dorsal and lumbar pain. Computed tomographic scan showed complete thrombosis of the false lumen at the stent-graft level and partial thrombosis below (Figure 1). Nevertheless, the thoracoabdominal aortic diameter had increased. We decided to perform regular surgery for treatment of the thoracoabdominal aneurysm. Femorofemoral 